\title{
Fiber fabry-pero micro pressure sensor
}

\author{
Xiaojing Yang ${ }^{1,2}$, Yiting Wang ${ }^{1,3}$ \\ 1.College of Computer and information engineering, Anyang Normal University, Anyang 455000 \\ 2. International WIC Institute, Beijing University of Technology, Beijing 100124
}

\begin{abstract}
A new fiber fabry-perot pressure sensor is designed. It is made by photolithography, silicon wafer etching, anode bonding, microelectromechanical system technology and so on. It is suitable for the pressure measurement of micro-pressure environment under harsh environment and small space. The structure of the transmitter and the way to make it are described in detail. The design smartly uses the Fiber optic flange, the optical fiber end face is parallel to the sensitive film, thus a high-quality Fabry-Pero-cavity is formed. The structure also contributes to the stability of the initial cavity, the sensor error is reduced. The experimental demodulation system is established, the pressure, temperature and other characteristics are tested in detail. The experiment result shows that within the pressure range of $0 \sim 0.1 \mathrm{MPa}$, the sensor has a good linearity, high repeatability and a sensitivity of $61.6 \mu \mathrm{m} / \mathrm{MPa}$.
\end{abstract}

Keywords: sensors; micro-pressure sensor; fiber optics sensor; Fabry-Perot; micro-electromechanical system technology

\section{Introduction}

Micropressure sensor is the most commonly used pressure sensor in industrial practice, widely used in various industrial automatic control environment, it is involved in oil pipelines, water and electricity, railway transportation, intelligent building, production of automatic control and machine tools and other industries[1.2]. But in the industrial security zone, against bad conditions, micro pressure leak in confined Spaces and test requirements for pipeline microflow have not been satisfied, need small size, high anti-interference, high sensitivity, micro pressure sensor products with wide range and not lose the precision. In recent years, the development direction of micropressure sensor is starting to shift to new materials, new mechanism and new structure, the process is being developed from silicon micropressure sensor $[3,4]$ to optical micropressure sensor.

Meanwhile, fiber fabry-perot(F-P) sensor is developing rapidly, it has been applied to severe electromagnetic interference and high temperature and other harsh conditions. The optical fiber F-P interferometric pressure sensor structure is simple, low cost, it is the most commonly used interferometric fiber pressure sensor. It not only has the characteristics of general optical fiber sensor good reliability, anti-electromagnetic interference, corrosion resistance, etc ,but also has the characteristics of high measure precision, high dynamic range, linearity and so on.

This paper designs and develops a fiber F-P micro pressure sensor, make full use of the advantages of optical fiber sensor, the disadvantages of traditional micro pressure sensor in small space and strong electromagnetic environment are overcome, and linearity is good, the sensitivity is high. The sensor is made of microelectromechanical system(MEMS) technology and suitable for mass serialproduction.

\section{2 sensor design}

The structure of the sensor is shown in figure 1, among them $a$ is the fiber optic flange, $b$ is the fiber and ceramic inserts, $\mathrm{c}$ is the glass ring, $\mathrm{d}$ is monocrystalline silicon piece, also called the pressure sensitive membrane of the sensor. The light travels vertically through the fiber, part of it is reflected off the fiber end, the other part passes through the air cavity, the silicon sensitive film is reflected back into the optical fiber, two beams of light interfere in the optical fiber, the F-P interference cavity was formed under the surface of the fiber optic end and the 
sensitive film. When external pressure changes, the sensitive membrane changes under the action of pressure, that changes the length of the F-P cavity, the phase of the reflection spectrum is changed. By means of the demodulation of its reflection spectra, you get the length of the F-P cavity.

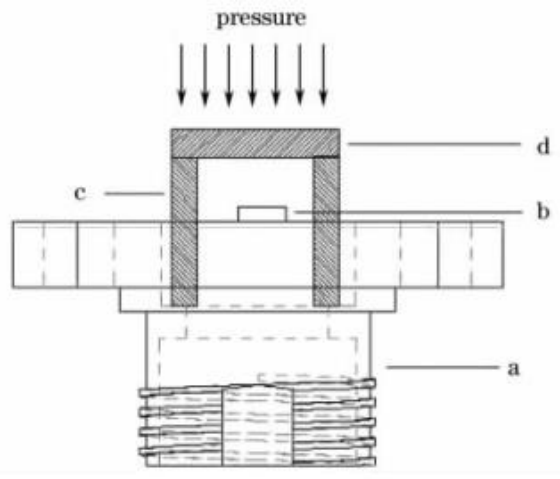

Figure 1 Schematic diagram of the structure

MEMS technology is used in the structure of sensor, the main features include lithography, silicon wafer etching and anode bonding, See figure 2. steps of:1) photoetching, is the geometric figure on the mask board, through photochemical reactions, transfer the pattern to a photoreceptor coated on a wafer. The process of coating, prebaking, exposure, developing and postbaking,ect, forming a photoresist pattern on a silicon wafer, as shown in figure2(a),(b);2) wet corrosion of silicon wafers coated with photoresist protection layer, use a certain concentration of $\mathrm{KOH}$ solution[9-10], the (100) wafers to silicon is etched into the desired shape and thickness, as shown in figure2(c), and degumming and surface cleaning processing, as shown in figure2(d);3) the obtained silicon wafers and pre-optical polishing, the long - precision controlled glass ring is used for the anode bonding, as shown in figure2(e), the bonding temperature selection is the key. The temperature is too low to reach the glass melting point, the temperature is too high because of the different thermal expansion coefficient, The stable work of the sensor is affected;4) the bonded silicon and glass rings are fixed on the FOCI fiber flange, finally insert the ceramic pin. The whole sensor is low cost and suitable for mass production.

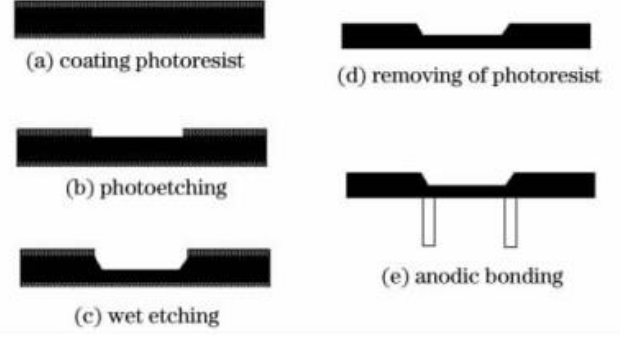

Figure 2 Sensor production process

In this sensor, the mechanical model of silicon sensitive film with glass ring bonding can be abstracted as radius $\mathrm{r}$, a round plate of surrounding solids, the surface is subjected to uniformly distributed pressure $\mathrm{P}$, then the relationship between the center deflection of silicon membrane and pressure $\mathrm{P}$ is

$\frac{\mathrm{Pr}^{4}}{E h^{4}}=\frac{16}{3\left(1-\mathrm{V}^{2}\right)} \frac{(Y)}{h}+\frac{7-V}{3(1-V)}\left(\frac{Y}{h}\right)^{3}$

The $\mathrm{h}$ is sensitive film thickness, $\mathrm{y}$ is the sensitive film center deflection. In general $(\mathrm{y}<\mathrm{h} / 2),(1)$ can be approximated to $\mathrm{A}$ linear equation:

$y=\frac{3 p r^{4}\left(1-V^{2}\right)}{16 E h^{3}}$

$\mathrm{P}$ is the external pressure, $\mathrm{r}$ is the sensitive membrane radius, $\mathrm{E}$ is young's modulus, $\mathrm{v}$ is poisson's ratio.For monocrystalline materials, $\mathrm{E}$ is $160 \mathrm{GPa}, \mathrm{v}$ is 0.22 . The assignment $r=3800 \mu \mathrm{m}, h=200 \mu \mathrm{m}$, apply pressure of $0.1 \mathrm{MPa}$, use software to simulate, the results are shown in figure 3 , different colors represent different degrees of deflection,the specific values are given by the ruler in the figure, the deflection of the circular film center is $5.9 \mu \mathrm{m}$.

\section{Analysis of experimental results}

The pressure test system of micropressure sensor is shown in figure 4,the broadband source with the optical fiber sensor Si720 is used as the light source of the sensor. The light passes through the coupler and divides into two equal paths, all the way as the incident light enter fiber F-P micro pressure sensor, the light reflected by the sensor passes through the same coupler into the sensing analyzer. The spectral signal of the reflected light is collected by the sensor analyzer to find the cavity length under certain pressure. Due to the membrane deflection and pressure are linear relationships, therefore, the corresponding 
pressure can be determined according to the change of lumen length.
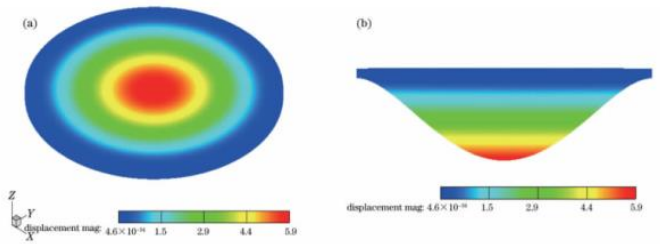

Figure 3 Simulation results of the sensitive membrance. (a)Front view; (b)side view

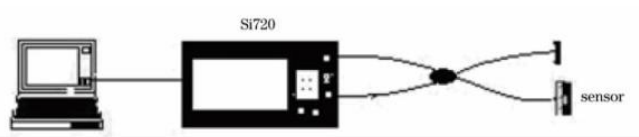

Figure 4 Pressure test system of the micro sensor

The reflectance spectra of the sensing analyzer are shown in fig.5.

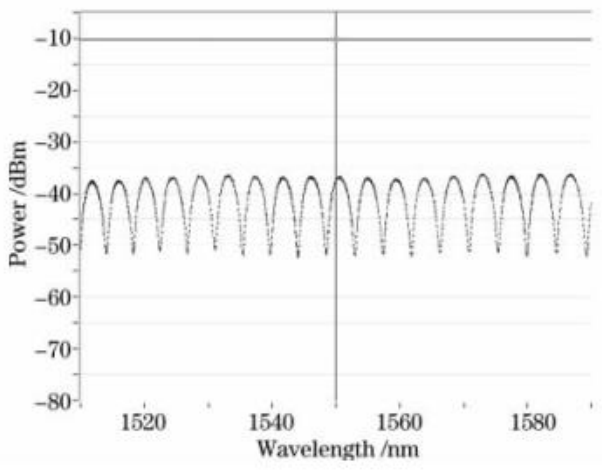

Figure 5 Reflectance spectra of micro pressure sensor

For the adjacent levels in the spectrum $\mathrm{m}, \mathrm{m}+1$, its spectral peak corresponds to the wavelength $\lambda_{m}, \lambda_{m+1}$ and cavity length $\mathrm{L}$. The following relationships exist:

$$
\begin{aligned}
& \frac{4 \pi L}{\lambda_{m}}=(2 m+1) \pi \\
& \frac{4 \pi L}{\lambda_{m+1}}=[2(m+1)+1] \pi
\end{aligned}
$$

F-P cavity length can be solved by (3), (4), We get the change in the cavity length, so let's figure out what the external pressure is.

The finished micro pressure sensor is marked with the YS-2.5 piston type pressure gauge, the pressure is uniformly increasing from 0 to $0.1 \mathrm{MPa}$. The initial cavity length of the micropressure sensor is $208.36 \mu \mathrm{m}$, the length of the lumen is calculated using Matlab software, the results are shown in figure 6, the lumen length fitting equation $\mathrm{G}=208.4+61.79 \mathrm{P}$, error of fitting 0.9999 , root-mean-square error 0.02033 , The sensitivity of the sensor is $61.6 \mu \mathrm{m} / \mathrm{MPa}$. The simulation results are shown in figure 3, external pressure change is 0.1 $\mathrm{MPa}$, deflection change of the circle membrane center is 5. $9 \mu m$, the experiment changed $6.1 \mu m$, the experimental results are consistent with the simulation results.

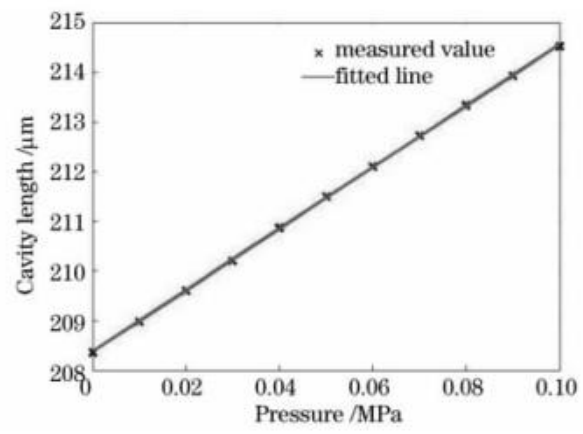

Figure 6 Relationship between pressure and cavity length of the micro pressure sensor

To test the delay performance of the sensor, increase the pressure from 0 to $0.1 \mathrm{MPa}$, and then it's uniformly down to zero, The contrast of lumen length in the process of lifting and lowering is shown in fig. 7. It can be seen that the delay effect of micro pressure sensor is very weak, the rise and fall curves almost coincide.

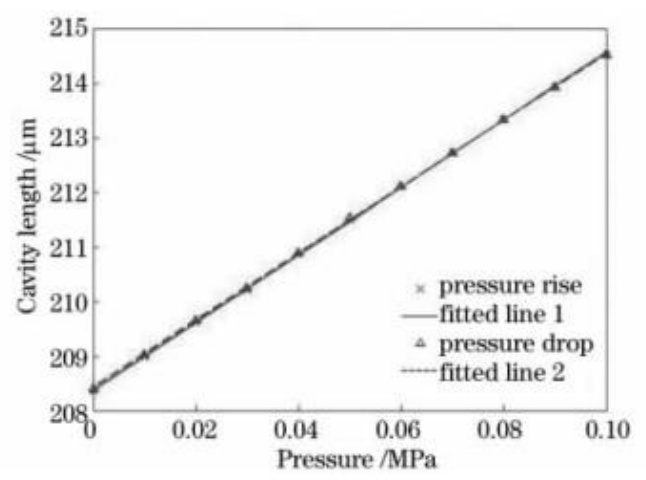

Figure 7 Cavity length changes during pressure rising and dropping

The experimental group also tested the repeatability of 
pressure sensors, the sensor was tested for four days in a row, the results are shown in table 1, the mean relative error of the calculation is $0.7 \%$,sensitivity to the sensor is minimal.

In addition, the temperature characteristic of the micro pressure transmitter is studied. Place a finished sensor in a high temperature test chamber, control the temperature rise from $10{ }^{\circ} \mathrm{C}$ to $65{ }^{\circ} \mathrm{C}$, the length of the lumen curve is shown in figure 8 . It can be seen that the cavity length of the sensor is linear with temperature change, the temperature sensitivity calculation value is $80 \mathrm{~nm} /{ }^{\circ} \mathrm{C}$. In stress tests, a good temperature linearity also facilitates temperature compensation for the sensor, to get accurate test results.

Table 1 Reperatablility of the sensor

\begin{tabular}{|c|c|c|c|c|c|c|c|c|c|c|c|}
\hline Pressure /MPa & 0 & 0,01 & 0,02 & 0,03 & 0,04 & 0,05 & 0,06 & 0.07 & 0,08 & 0,09 & 0,10 \\
\hline list day & $-0,0002$ & 0,0101 & 0.0201 & 0.0298 & 0.0405 & 0.0508 & 0,0606 & 0,0706 & $0,0800^{\circ}$ & 0,0903 & 0,1000 \\
\hline 2nd day & 0,0005 & 0,0106 & 0,0209 & $0,0,0,05$ & 0,0409 & $0,0,508$ & 0,0608 & 0,0707 & $0,0800^{2}$ & 0,0901 & 0,0999 \\
\hline $3 x d d a$ & $=0,000 \%$ & 0,0099 & 0,0199 & 0,0288 & $0,040,3$ & $0,0,007$ & 0,0606 & 0,0705 & 0,0805 & 0,0900 & 0,0997 \\
\hline 4th dy & 0,0000 & 0,0101 & 0.0202 & 0,0299 & $0,040,3$ & 0.0506 & $0.0600^{\circ}$ & 0.0706 & 0,0807 & 0.0899 & 0,0996 \\
\hline
\end{tabular}

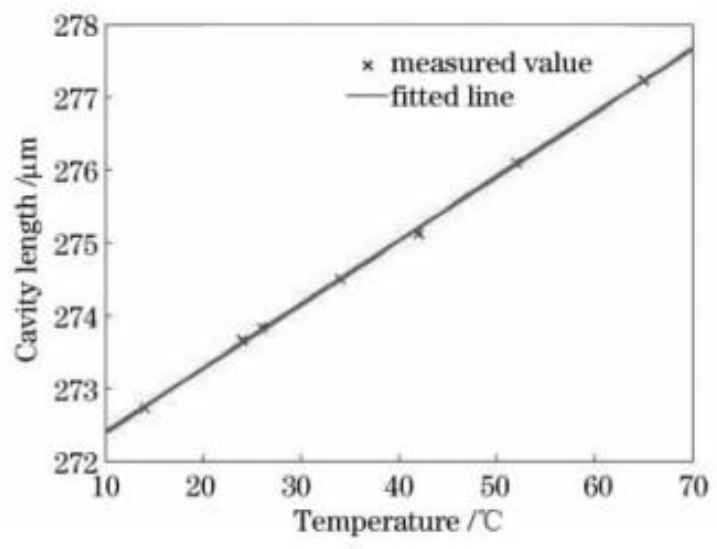

Figure 8 Temperature characteristic of the sensor

\section{Conclusions}

The design and manufacture of optical fiber F-P micro pressure sensor, a number of tests have been carried out. The results show that the micro-pressure sensor is stable, good hysteresis, high sensitivity, and it is easy to draw materials, Compact construction, the advantages of low production cost, suitable for mass production.

\section{References:}

1 Gregory T A Kovacs.Micromachined Transducers:Sourcebook [M].Boston:WCB, 1998.
2 Minxin Zhou,Qingan Huang,Ming Qin.Modeling,design and fabrication of a triple-layered capacitive pressure sensor[J].Sensors and Actuators A:physical,2005,117(1):71-81.

3 E G Bakhoum,M H Cheng.Capacitive pressure sensor with very large dynamic range [J].IEEE Transactions on Components and Packaging,2010,33,79-83.

4 Zhenhai Chen, R C Luo.Design and implementation of capacitive proximity sensor using microelectromechanical systems technology[J].IEEE Transactions on Industrial Electronics,2002,45:886-894.

5 Yang Chundi,Wang Ming,Ge Yixian,et al..A miniature extrinsic fiber Fabry-Perot pressure sensor[J].Acta Optica Sinica,2011,30(5):1358-1361.

6 Wang Lihua,Wang Ming,Rong Hua,et al..Design and simulation of miniature shear stress sensor based on Fabry-Perot interference[J].Acta Optica Sinica,2012.32(sl):s128003.

7 Ni Xiaoqi,Wang Ming,Chen Xunxing,et al..Wavelength multiplexing of optical fiber microelectromechanical system Fabry-Perot pressure sensor[J].Acta Optica Sinica,2007,27(5):0776-0780.

$8 \mathrm{Ke}$ Tao,Zhu Tao,Rao Yunjiang,et al..Accelerometer based on all-fiber Fabry-Perot interferometer formed by hollow-core photonic crystal fiber[J].Chinese J Lasers,2010,37(1):171-175.

9 Yanfeng Jiang,Qingan Huang.A physical model for silicon anisotropic chemical etching $[\mathrm{J}]$.Seminconductor Science and Technology,2005,20(6):524-531.

10Nacer Eddine Demagh,Assia Guessoum,Hind Aissat,Chemical etching of concave cone fibre ends for core fibre alignment[J].Meas Sci Technol,2006,17:119-122.

11Gregory T A Kovacs.Micromachined Transducers Sourcebook[M].Zhang Wendong et al.Transl.Beijing:Science Press,2003.181. 